Available online at GSC Online Press Directory

GSC Advanced Research and Reviews e-ISSN: 2582-4597, CODEN (USA): GARRC2

Journal homepage: https://www.gsconlinepress.com/journals/gscarr

(REVIEW ARTICLE)

\title{
The fourth transition, zoonosis and coronavirus
}

\author{
Jessica Pérez-Nava ${ }^{1}$, Rocío Pérez-Terrón ${ }^{2}$ and Antonio Rivera ${ }^{3, *}$ \\ ${ }_{1}$ Posgrado en Ciencias Ambientales, Instituto de Ciencias de la Benemérita Universidad Autónoma de Puebla, México. \\ 2 Facultad de Ciencias Biológicas de la Benemérita Universidad Autónoma de Puebla, México. \\ ${ }^{3}$ Centro de Investigaciones en Ciencias Microbiológicas, Instituto de Ciencias de la Benemérita Universidad Autónoma de \\ Puebla, México.
}

Publication history: Received on 13 March 2020; revised on 19 March 2020; accepted on 20 March 2020

Article DOI: https://doi.org/10.30574/gscarr.2020.2.3.0021

\begin{abstract}
A mini review of the situation regarding the presence of the coronavirus and its possible zoonotic origin is presented, in addition to commenting on the transitions of emerging diseases that have occurred throughout history and the importance they have had in society.
\end{abstract}

Keywords: Globalization; Emerging diseases; Fragmented landscapes; Coronavirus; Bat

\section{Introduction}

Some species of microorganisms can infect different kinds of hosts, an adaptive capacity that facilitates the transmission, colonization and infection of different species of hosts. Around 40\% of microbial pathogens with three or more nonhuman hosts are considered emerging, and the ability to colonize and infect a greater number of different types of hosts is associated with a greater chance of success in colonization and infection of the human. For example, the coronavirus associated with severe acute respiratory syndrome is of zoonotic origin, and can infect a wide variety of hosts and has recently appeared in humans [1].

Human societies have experienced important transitions throughout their history that have affected the patterns of acquisition and dissemination of infectious diseases. Four transitions in the emergence of infectious diseases have been identified, the first transition in prehistoric times gave rise to the first epidemic infections. These infections arose after the advent of agriculture when nomadic peoples became societies based on agricultural activities that developed more populated communities [2,3]. The second transition was in the old age when Eurasian civilizations came into contact with each other due to commercial and military activities, exchanging their reserves of infections and vectors [4]. The third transition occurs in the modern age and accompanies global exploration and colonization by Europeans [5]. Today we are living the fourth transition (globalization), the factors that influence the emergence of infectious diseases being diverse, and most depend on human activities. In addition, from the capacity of microbial adaptation to changes in their environment, the main determinants in the emergence of emerging diseases have been analyzed, the most important being economic impoverishment, migrations, use of pesticides and antibiotics, changes in behavior human, global warming and changes in decision-making schemes in public health. Without ruling out that the human ecological impact has consequences since it can accelerate the evolutionary changes in the species, particularly in those microorganisms that cause diseases $[6,7]$.

From the outbreak of severe acute respiratory syndrome (SARS) 18 years ago, several SARS-related coronaviruses (SARSr-CoV) were described in their natural reservoir, bats. Different studies have described that some bat SARSr-CoV have the ability to infect humans. Therefore, work has been done on the identification and characterization of the new

\footnotetext{
${ }^{*}$ Corresponding author: Antonio Rivera
}

Copyright (C) 2020 Author(s) retain the copyright of this article. This article is published under the terms of the Creative Commons Attribution Liscense 4.0. 
coronavirus (2019-nCoV), which caused acute respiratory syndrome in humans in Wuhan, China. The epidemic, which began last December, caused 2,794 infections, including 80 deaths before January 26, 2020. Work was done to obtain complete genomic sequences from five patients at an early stage of the outbreak. The sequences were almost identical and share a sequence identity of $79.6 \%$ with the SARS-CoV. In addition, $2019-n C o V$ was shown to be $96 \%$ identical to the genome of a bat coronavirus. Protein sequence analysis by pairs of seven conserved non-structural protein domains showed that this virus belongs to the SARSr-CoV species. In addition, the 2019-nCoV virus isolated from the bronchoalveolar lavage fluid of a critical patient was neutralized with sera from several patients. It has been confirmed that 2019-nCoV uses the same cellular access receptor (angiotensin II converting enzyme (ACE2)) as SARS-CoV [8].

Emerging infectious diseases, such as severe acute respiratory syndrome (SARS) and Zika virus disease, represent a major threat to public health. Despite intense research efforts, how, when and where new diseases appear remain a source of considerable uncertainty. A serious respiratory illness was recently reported in Wuhan, Hubei Province, China. As of January 25, 2020, at least 1,975 cases had been reported since the first patient was hospitalized on December 12, 2019. Epidemiological investigations have suggested that the outbreak was associated with a seafood market in Wuhan. Here we study a single patient who worked in the market and who entered the Wuhan Central Hospital on December 26, 2019 while experiencing a severe respiratory syndrome that included fever, dizziness and cough. Sequencing of metagenomic RNA from a sample of bronchoalveolar lavage fluid from the patient identified a new strain of RNA virus from the Coronaviridae family, here referred to as coronavirus WH-Human 1 (and is also known as 2019-nCoV). Phylogenetic analysis of the complete viral genome $(29,903$ nucleotides) revealed that the virus was more closely related (nucleotide similarity of $89.1 \%$ ) with a coronavirus group similar to SARS (genus Betacoronavirus, subgenus sarbecovirus) that had previously been found in bats in China. This outbreak highlights the continued ability of viral propagation of animals to cause serious diseases in humans [9].

In an investigation conducted in Yunnan Province, Guangxi and Guangdong, residents were included, from whom data were collected through ethnographic interviews and field observations, coding and thematically analyzed to identify risk and protective factors for disease occurrence zoonotics at individual and community levels.

Frequent interactions between humans and animals and low levels of environmental biosecurity in local communities were identified as risks for the occurrence of zoonotic diseases. The relationship between zoonotic risk and human behavior, the environment and policies in rural communities in southern China was also explored. It is suggested to identify the key behavioral risk factors that may be subject to the development of personalized risk mitigation strategies to reduce the threat of new zoonoses. In addition to the urgent need to establish strategies to mitigate the risk of occurrence of zoonotic diseases in southern China, where pathogens with zoonotic potential are known to circulate in wild animal populations. However, the risk factors that lead to the emergency are poorly understood, which presents a challenge in the appropriate development of mitigation strategies for local communities [10].

The ecological and phylogenetic analysis of the interactions between parasites and hosts can integrate the influence of the environment on the distribution of pathogens on temporal and spatial scales, as well as different levels of biological and taxonomic organization.

The relationships between host diversity and that of four viral groups associated with bats in Mexico have been examined [11], demonstrating that the quality and abiotic characteristics of the habitat determine the viral diversity in landscapes dominated by human activities. In addition, where the host replacement component represents an unexplored dimension with a high potential to estimate viral diversity, especially in fragmented landscapes. Quantifying the turnover of viral communities associated with bats and monitoring the potential richness of viruses in wildlife and their relationship with biodiversity, a necessary issue to understand how changes in the function of ecosystems and anthropogenic activities promote the onset of diseases.

In conclusion, the zoonotic transmission is suspected as the route of disease origin, analyses predict bats as the most probable source of 2019-nCoV though further investigations need to confirm the origin of the novel virus.

\section{Compliance with ethical standards}

\section{Acknowledgments}

All Authors listed have made a substantial and intellectual contribution to the work, and approved it for publication. 


\section{Disclosure of conflict of interest}

The author declares no conflict of interest.

\section{References}

[1] Perris JSM, Yuen KY, Osterhaus ADME and Stöhr K. (2003). Current concepts: the severe acute respiratory syndrome. N Engl J Med., 349, 2431-2441.

[2] Weiss RA and McMichael AJ. (2004). Social and environmental risk factors in the emergence of infectious diseases. Nat Med., 10, S70-S76.

[3] Diamond J. (2002). Evolution, consequences and future of plant and animal domestication. Nature, 418, 700-707.

[4] Morens DM and Littman RJ. (1992). Epidemiology of the plague of Athens. Trans Am Philol Assoc, 122, 271-304.

[5] Duffy J. (1951). Smallpox and the Indians in the American colonies. Bull Hist Med, 25, 324-341.

[6] Feldman H, Club M, Jones S, Dick D, Garbutt M and Grolla A. (2002). Emerging and re-emerging infectious diseases. Med Microbiol Immunol, 191, 63-74.

[7] Palumbi SR. (2001). Humans as the world's greatest evolutionary force. Science, 293, 1786-1790.

[8] Zhou P, Yang X, Wang X, Hu B, Zhang L, Zhang W, Si H, Zhu Y, Li B, Huang C, Chen H, Chen J, Luo Y, Guo H, Jiang R, Liu M, Chen Y, Shen X, Wang X, Zheng X, Zhao K, Chen Q, Deng F, Liu L, Yan B, Zhan F, Wang Y, Xiao G and Shi Z. (2020). A pneumonia outbreak associated with a new coronavirus of probable bat origin. Nature.

[9] Wu F, Zhao S, Yu B, Chen Y, Wang W, Song Z, Hu Y, Tao Z, Tian J, Pei Y, Yuan M, Zhang Y, Dai F, Liu Y, Wang Q, Zheng J, Xu L, Holmes E and Zhang Y. (2020). A new coronavirus associated with human respiratory disease in China. Nature.

[10] Li H, Zhu G, Zhang Y, Zhang L, Hagan E, Martínez S, Chmura A, Francisco L, Tai H, Miller M and Daszak P. (2020). A qualitative study of zoonotic risk factors among rural communities in southern China. Int Health, 12, 77-85.

[11] Rico C0, Ojeda FR, Sotomayor BJ, Zambrana-Torrelio C, Loza RE, Alonso AA and Suzán G. (2015). Diversidad viral de comunidades de murciélagos en paisajes transformados de México. Vet México OA, 2, 1-23.

\section{How to cite this article}

Pérez-Nava J, Pérez-Terrón R and Rivera A. (2020). The fourth transition, zoonosis and coronavirus. GSC Advanced Research and Reviews, 2(3), 49-51. 